\title{
EL PROGRAMA INTEGRAL PARA ALTAS CAPACIDADES (PIPAC): 18 AÑOS DE EXPERIENCIA
}

\author{
Manuela Rodríguez, Dorta* \\ Triana Aguirre Delgado** \\ Africa Borges***
}

\begin{abstract}
RESUMEN: El alumnado más capaz, debido a sus características diferenciales, requiere de una respuesta educativa específica. Sin embargo, aún queda camino por recorrer en este sentido, ya que su detección e identificación está muy por debajo de los estándares señalados por los expertos y la respuesta educativa necesaria sigue siendo insuficiente. Por tanto, es imprescindible diseñar programas, tanto intra como extraescolares que, además, incorporen a la familia, pues son uno de los principales agentes de detección y tienen un importante papel en la respuesta educativa de sus hijos. Así, en la Universidad de La Laguna se pone en marcha el Programa Integral para Altas Capacidades (PIPAC) cuya finalidad es contribuir al desarrollo de este alumnado a nivel cognitivo, socioafectivo y comportamental, a lo largo de diversos cursos e integrando en él a la familia. Este programa ha ido evolucionando y adaptándose a las características y necesidades de los participantes, así como a las circunstancias derivadas de la actual situación de crisis sanitaria. En el presente artículo se muestran las características y fortalezas que favorecen el éxito del programa, así como su reciente adaptación al formato online y la contribución de la Universidad de La Laguna a las altas capacidades intelectuales.
\end{abstract}

PALABRAS CLAVE: Respuesta educativa; Programas específicos; Evaluación.

\section{O PROGRAMA INTEGRAL PARA ALTAS CAPACIDADES (PIPAC): 18 ANOS DE EXPERIENCIA}

RESUMO: Os alunos com mais capacidades, pelas suas características diferenciadoras, requerem uma resposta educativa específica. No entanto, há ainda um longo caminho a percorrer nesse sentido, visto que a sua detecção e identificação se encontram muito aquém dos padrões indicados pelos especialistas, sendo a devida resposta educativa ainda insuficiente. Portanto, é imprescindível o desenho de programas, a nível curricular e extracurricular, que incluam também a família, uma vez que ela é um dos principais agentes de detecção e tem um papel importante na resposta educacional aos filhos. Assim, na Universidade de La Laguna é aplicado o Programa Integral de Altas Capacidades (PIPAC), cujo objetivo é contribuir para o desenvolvimento desses alunos em nível cognitivo, socioafetivo e comportamental, ao longo de vários anos escolares e integrando a família. Este programa tem evoluído e tem-se adaptado às características e necessidades dos participantes, bem como às circunstâncias decorrentes da atual situação de crise de saúde. Este artigo evidencia as características e potencialidades que favorecem o sucesso do PIPAC, bem como sua recente adaptação ao formato online e a contribuição da Universidade de La Laguna para as altas capacidades intelectuais.

PALAVRAS-CHAVE: Resposta educativa; Programas específicos; Avaliação.

\footnotetext{
* Licenciada y Doctora en Psicología y Máster Universitario en Psicología de la Educación, Universidad de La Laguna, Espanha. Grupo de Investigación Aplicada en Ciencias del Comportamiento (GIACCo), https://orcid.org/0000-00032979-7247, manuela@gtisd.net

${ }^{* *}$ Graduada en Trabajo Social y Máster Universitario en Mediación Familiar y Sociocomunitaria y en Intervención Familiar, Universidad de La Laguna, Espanha. Grupo de Investigación Aplicada en Ciencias del Comportamiento (GIACCo), https://orcid.org/0000-0002-2110-1551, taguirre@ull.edu.es

${ }^{* * *}$ Catedrática de Psicología, Universidad de La Laguna, Espanha. Grupo de Investigación Aplicada en Ciencias del Comportamiento (GIACCo), https://orcid.org/0000-0001-8267-4401, aborges@ull.edu.es
} 


\title{
THE INTEGRAL PROGRAM FOR GIFTEDNESS (PIPAC):
} 18 YEARS OF EXPERIENCE

\begin{abstract}
The most able students, due to their differential characteristics, require a specific educational response. However, there is still some way to go in this respect. The detection and identification of these students is still far below the standards indicated by experts and the educational response is still insufficient. Therefore, it is essential to design programmes, both intra and extracurricular, that also involve the family, as they are one of the main detection agents and play an important role in the educational response of their children. Thus, at the University of La Laguna has set up the Comprehensive the Comprehensive Programme for High Abilities (PIPAC), whose main objective is to contribute to the development of these students at a cognitive, socioemotional and behavioral level, throughout various courses and integrating the family. This programme has evolved and adapted to the characteristics and needs of the participants, as well as to the circumstances arising from the current health crisis. This article shows the characteristics and strengths that support the success of the programme, as well as its recent adaptation to the online format and the contribution of the University of La Laguna to high intellectual abilities.
\end{abstract}

KEYWORDS: Educational response; Specific programs; Evaluation.

\section{Necesidad de respuesta educativa específica para el alumnado de altas capacidades}

La literatura científica especializada en el alumnado más capaz ha puesto de manifiesto que tiene características cognitivas diferenciales, tanto a nivel de funciones ejecutivas, como por ejemplo mayor memoria de trabajo (RODRÍGUEZ-NAVEIRAS et al., 2019), flexibilidad cognitiva (ARANCIBIA et al., 2016), mayor manejo de información que procesan con más velocidad (STEINER; CARR, 2003), habilidades metacognitivas (SASTRE-RIBA, 2012; STEINER; CARR, 2003; VAIVRE-DOURET, 2011) o capacidad de resolución de problemas (SASTRE-RIBA, 2008).

Estas diferencias exigen una respuesta educativa específica, para lograr el máximo de sus potencialidades. No obstante, queda un amplio camino por recorrer aún porque, aunque muchos países, entre ellos España, incorporan estas necesidades en su normativa tanto estatal como autonómica (COMES et al., 2009; QUINTERO et al., 2021), la realidad es que el primer proceso necesario para responder a las necesidades educativas de este alumnado empieza con la identificación del alumnado de altas capacidades y talento. En este sentido, si bien en España se ha incrementado bastante el porcentaje de identificados, pasando de un 0,09\% en el curso 2011/2012 a un 0,43 \% en las últimas estadísticas dadas por el Ministerio de Educación y Formación profesional, esto es, las relativas al curso 2018/2019 $\mathrm{Y}$, lo que es curioso, la proporción de alumnado identificado no es homogénea en todas las comunidades autónomas españolas, dándose incluso importantes oscilaciones dentro de la misma comunidad autónoma, a pesar de que rige en todas las provincias incluidas la misma legislación (QUINTERO et al., 2021). A pesar del notable incremento del número de estudiantes diagnosticados, sigue estando muy por debajo de al menos el 10\% que se espera que sean talentos o altas capacidades en la previsión de los expertos (HERNÁNDEZ-TORRANO; GUTIÉRREZ SÁNCHEZ, 2014). 
Por otra parte, una vez hecho el diagnóstico y cuando ya se sabe que el o la estudiante tiene altas capacidades o algún tipo de talento, el siguiente paso es dar la necesaria respuesta educativa, preferentemente intraescolar. De nuevo los datos muestran que queda un largo camino que recorrer: en un reciente estudio donde se entrevistó a progenitores de alumnado de altas capacidades (RODRÍGUEZNAVEIRAS et al., 2019), solo un $24 \%$ recibían programas intraescolares para altas capacidades.

Por tanto, es imprescindible diseñar programas específicos para este alumnado, tanto intra como extraescolares. Si bien es preciso que la respuesta educativa se dé dentro del aula, para el adecuado desarrollo de las potencialidades de este alumnado, y evitar problemas importantes derivados de un inadecuado proceso educativo, tales como aburrimiento, desmotivación, falta de desarrollo de hábitos de estudio, con importantes consecuencias en el currículo académico, la realidad es que dentro de la escuela no se atiende a todo el alumnado más capaz, lo que obliga a ofrecer programas extraescolares, que permitan paliar este problema.

Si bien la literatura especializada es más dispar en cuanto a características y ajuste socioafectivo, no pudiendo mantenerse en la actualidad que este colectivo tenga más problemas de ajuste personal y social debido a su mayor capacidad intelectual (BORGES et al., 2008; BORGES et al., 2011; MATÍAS RANCEL, 2020), sí que se señalan la existencia de algunas características, como el perfeccionismo, un sentido crítico importante con respecto a sí mismos y a los demás, así como tendencia a presentar altas expectativas (ARETXAGA et al., 2013; PEÑAS, 2006), lo que puede conducir a que puedan sentir, en ocasiones una cierta sensación de fracaso, cuando sus acciones no alcanzan sus expectativas. Además, tienen un locus de control interno para atribuirse los éxitos obtenidos, pero externo a la hora de achacar los fracasos a otras personas (OLSZEWSKI-KUBILIUS; KULIEKE; KRASNEY, 1988). Posiblemente debido a este perfeccionismo y altas expectativas, los niños y niñas con altas capacidades presentan una baja tolerancia a la frustración (ARETXAGA et al., 2013; ALENCAR, 2008), que es preciso trabajar directamente (GÓMEZ, 2020; PÉREZ-HERNÁNDEZ; RODRÍGUEZ-NAVEIRAS, 2019). Otra dificultad es que son poco proclives a trabajar de forma grupal (RODRÍGUEZ-NAVEIRAS et al., 2015), siendo una realidad que con frecuencia es obligado trabajar en equipo y deben estar preparados para ello.

Por otra parte, la intervención con este alumnado debe de incorporar a la familia, pues no en vano diversos modelos explicativos de las altas capacidades la incluyen dentro del entorno social donde se desenvuelven (GAGNÉ, 2009; TANNENBAUM, 1986; VAN BOXTEL; MÖNKS, 1988). Además, son uno de los principales agentes de detección (BORGES; HERNÁNDEZ-JORGE, 2018), que supone el paso previo para la identificación, jugando también un papel importante en la identificación en algunas de las Comunidades Autónomas, como por ejemplo en Canarias (COMES et al., 2008). Por otra parte, cuando la identificación se confirma, los progenitores no siempre lo reciben como un privilegio, sino que pueden darse reacciones adversas, al pensar que va unido a problemas, tanto de ajuste como de adaptación social (PÉREZ, 2004), tal vez influidos por los perniciosos mitos que aún tienen vigencia sobre las altas capacidades (PÉREZ et al., 2020). Finalmente, los progenitores tienen un importante papel 
en la respuesta educativa de sus hijos, pues suelen verse en la necesidad de buscar formas alternativas para que sus hijos o hijas reciban la formación que precisan, cuando la institución educativa no da solución a sus necesidades (FLORES et al., 2020).

\section{Surgimiento y evolución del Programa Integral para Altas Capacidades (PIPAC)}

Ante la necesidad de dar una respuesta educativa al alumnado de altas capacidades, en el curso 2002-2003 se forma en la Universidad de La Laguna el Grupo de Trabajo e Investigación en Superdotación, empezando su andadura formándose en altas capacidades, e iniciando el conocimiento de este colectivo con una investigación cualitativa, dando lugar a tesinas y trabajos de investigación en los programas de doctorado (DÍAZ CABRERA, 2004; RODRÍGUEZ-NAVEIRAS, 2006). A partir de ahí, se diseña el programa, que se pone en práctica en el curso 2003-2004.

El Programa Integral para Altas Capacidades (PIPAC) (BORGES; RODRÍGUEZ-NAVEIRAS; RODRÍGUEZ-DORTA, 2018; RODRÍGUEZ-NAVEIRAS et al., 2015; RODRÍGUEZ-NAVEIRAS; BORGES, 2020) tiene como objetivo principal contribuir al desarrollo de este alumnado desde una perspectiva integral.

Es planteado como un programa de intervención psicoeducativa que pone el foco de atención en el abordaje de aspectos socioafectivos. Se fundamenta en un modelo evolutivo, en cuanto atiende a los participantes a lo largo de diversos cursos, y ambiental, teniendo en cuenta la influencia del contexto que les rodea. Así, atiende a escolares de Altas Capacidades Intelectuales de Educación Primaria y Secundaria a través del subprograma Descubriéndonos, y a sus progenitores por medio del subprograma Encuentros.

A lo largo de estos dieciocho años de historia, el PIPAC ha ido evolucionando y adaptándose a las características y necesidades de los participantes, así como a las circunstancias derivadas de la actual situación de crisis sanitaria.

El subprograma Descubriéndonos se implementa de forma quincenal. En un principio acoge a niños y niñas de entre 3 y 12 años, que, para una mejor atención y organización, se dividen en tres niveles de edad: (a) nivel 1 de 3 a 6 años; (b) nivel 2 de 7 a 9 años; y (c) nivel 3 de 10 y 12 años. Sin embargo, debido al aumento de niños y niñas con edades correspondientes al nivel 2, desde el curso 2015-2016, dicho nivel se desdobló en dos grupos, por lo que se pasa a tener 4 niveles. Más adelante, curso 2019-2020, y teniendo en cuenta la continuidad de los participantes en el programa a lo largo de los diferentes cursos, se amplía el límite de edad hasta los 14 años y se integra un nuevo nivel, nivel 5. Además, como ya se ha comentado, en él se integran a los hermanos y las hermanas no sometidos a diagnóstico, con un doble objetivo: evitar la diferenciación y etiquetación en el seno de la familia e incluir e implicar en el programa a todo el contexto familiar del niño o la niña con diagnóstico.

Las sesiones con cada uno de los grupos están dirigidas por dos educadores, al menos, educador principal y básico. El primero, quien cuenta con amplia formación y dilatada experiencia en éste, tiene el 
rol de implementar y dirigir las sesiones de intervención. El segundo, quien también cuenta con formación, pero con menor experiencia, tiene un papel de ayuda a las labores que ejerce el primero. Además, el programa acoge a estudiantes de Grado y Máster en prácticas. Éstos se integran en los grupos como educadores en formación, cuyo papel es en, un principio, de observador y conforme avanza el curso, bajo la orientación y supervisión del educador principal, comienza a tener un papel más activo en las sesiones, diseñando y llevando a cabo diferentes actividades.

Los contenidos se abordan desde un ambiente de seguridad distinto al escolar en el que los participantes se sientan cómodos. Se diseñan a través de una planificación previa, aunque flexible, adaptándose en función de las características y necesidades del grupo. La metodología empleada es lúdica y experiencial, para captar y mantener la atención de los participantes. Así, en las diferentes sesiones, se llevan a cabo diversas actividades con objetivos muy claros, de corta duración, se ponen ejemplos de situaciones reales, se resuelven ejercicios en grupo, se les da la oportunidad de descubrir por sí mismos la mejor solución y, sobre todo, con los más pequeños, se emplea material llamativo que se puedan manipular.

Con respecto a los aspectos que se plantean, hay que señalar que, desde su inicio, aunque se abordan aspectos cognitivos, el trabajo se centra en aspectos de carácter socioafectivo, siendo el principal foco de atención. Así, en el primer trimestre, se desarrollan aspectos de tipo intrapersonal, con el objetivo de fomentar el conocimiento de sí mismo (autoestima, autoconcepto, autocontrol...). En el segundo trimestre, se trabajan aspectos interpersonales para la adquisición de habilidades sociales que favorezcan una adecuada interacción con los demás (escucha activa, empatía, asertividad). Finalmente, y aunque se trabaja de forma transversal a lo largo de todo el curso, en el tercer trimestre la intervención pone énfasis en el trabajo en equipo (organización dentro de un grupo, distribución de roles, gestión de conflictos, toma de decisiones en grupo).

Además, a lo largo de los cursos se incluyen actividades concretas para el trabajo en mayor profundidad de los diferentes aspectos socioafectivos programados. Así, con el objetivo de un mayor desarrollo de las habilidades de interacción con los demás, cohesionar a los participantes de los diferentes niveles y facilitar un mayor conocimiento y mejor relación entre ellos, en el curso 2015-2016, se incorpora la actividad "Visita a los vecinos". Ésta se lleva a cabo por medio de diferentes encuentros entre los distintos niveles. Se inicia con primer encuentro, donde, con la finalidad de que los participantes de distintos niveles se conozcan y se relacionen entre sí, éstos se presentan a sus compañeros y exponen en qué están trabajando. Y un segundo encuentro donde, con el objetivo de que los niños y las niñas aprendan a trabajar con otros compañeros de edades distintas, éstos participan en una actividad en conjunto. También, y como un Trabajo Final de Grado en un curso (PÉREZ; RODRÍGUEZ-NAVEIRAS, 2019) y como Trabajo Final de Máster en el siguiente (GÓMEZ, 2020), se lleva a cabo un subprograma de intervención específico para la mejora de la tolerancia a la frustración. 
Con respecto a los aspectos cognitivos, comienzan a tener a lo largo de las diferentes ediciones una mayor relevancia, destinando más tiempo de las sesiones al trabajo de los mismos. Así, se incluyen diferentes actividades de este tipo, como tareas de estimulación de la memoria, la atención y la concentración, a través de fichas y actividades interactivas entre los niños y las niñas, trabajando, además, con ellas de forma paralela la tolerancia a la frustración y la persistencia en la tarea; experimentos científicos con los que se pretende fomentar el interés y gusto por la ciencia, estimular la curiosidad e inquietudes intelectuales; la actividad "El Rincón del Interés", donde los participantes han de realizar presentaciones sobre un tema dado, siendo su objetivo trabajar el manejo y organización de la información y la expresión oral, así como profundizar en el conocimiento de diversas temáticas, lo que a su vez hace que se adquieran hábitos y técnicas de estudio. Además, se organizan para culminar como trabajo de fin de trimestre (SANCHO et al., 2017).

Es importante señalar que las actividades de carácter cognitivo para fomentar la atención, la concentración y la memoria tienen, dentro del programa, una doble vertiente. Por una parte, permiten el trabajo de dichos aspectos y, por otra, suponen una estrategia de control y contraste estimular, ya que al ser presentados como retos y como aspectos novedosos en el transcurso de las sesiones, permiten reconducir y centrar la atención de los participantes y así poder retomar de nuevo el trabajo socioafectivo con un mayor nivel de atención y motivación.

Además, hay que destacar que los trabajos finales de trimestre en un principio se plantearon para el fomento del trabajo cooperativo. Así, se desarrolla un trabajo final para cada trimestre sobre un tema elegido por los propios participantes o establecido por los educadores, siendo presentados al final de cada uno al resto de compañeros y grupo de familias y educadores. Sin embargo, esta actividad comienza a tomar una dimensión más amplia, trabajando en mayor profundidad los temas elegidos durante más tiempo, esto es a lo largo de todo el curso, por lo que se convierten en proyectos de investigación grupal dando lugar a un producto final. Con ellos se dirige el foco de atención al fomento y desarrollo de aspectos más cognitivos, como habilidades de investigación, búsqueda de información y organización de la misma, sin olvidar que los participantes desarrollen la responsabilidad por la tarea, la persistencia en la misma, adquisición de hábitos y técnicas de estudio y habilidades para el trabajo en equipo. Así, por ejemplo, en el curso 2016-2017, se plantea a los participantes de los diferentes grupos trabajar en el desarrollo de una idea que permita mejorar la vida de las personas en su contexto más cercano. El producto final de este proyecto se materializa en un corto que expresa de forma gráfica la idea y pretende crear consciencia social sobre el tema abordado (CADENAS et al., 2017). En el curso 2017-2018, se les plantea trabajar distintos aspectos del agua en Canarias. En el 2018-2019 el tema de partida en esta ocasión es el arte y la creatividad (CURBELO; MONTERO, 2020). Durante el presente curso, el tema monográfico que han ido desarrollando es la utilización práctica de materiales reciclados.

Otro de los aspectos cognitivos abordados es el trabajo de la caligrafía. Este se plantea para dar respuesta al bajo rendimiento que este alumnado puede mostrar en dicho aspecto, el cual se manifiesta 
como consecuencia de la disincronía que puede tener lugar entre el desarrollo de la capacidad intelectual y el desarrollo de la capacidad motriz. La caligrafía es abordada a través de diferentes actividades. Así, una de las actividades llevadas a cabo para trabajarla es "Escribiendo a un amigo", donde los participantes se cartean con los/as compañeros/as de otros grupos, desarrollando en la quinceava edición del programa una intervención para la mejora de la caligrafía a través de las cartas entre los participantes, que supuso otro Trabajo Final de Grado (MARÍN; RODRÍGUEZ; BORGES, 2020).

El PIPAC no solo atiende a escolares con Altas Capacidades, sino que, además, tiene en cuenta el importante papel que juega la familia en el desarrollo infantil y que éste es de especial relevancia en el caso de niños y niñas con Altas Capacidades Intelectuales para el desarrollo de determinados aspectos (STOEGER et al., 2014). Por ello surge el subprograma Encuentros (BORGES et al., 2016) que está dirigido a padres y madres de niños y niñas participantes en el programa. Éste permite implicar a todo el contexto familiar y así dar una respuesta más integral.

La metodología de trabajo de este subprograma es experiencial, trabajando de forma individual y en pequeños grupos, orientados por los educadores, distintas situaciones de la vida cotidiana y debatiéndose posteriormente en grupo. Al mismo tiempo, los educadores abordan aspectos conceptuales que apoyan y refuerzan la práctica.

Comienza marcándose como objetivo principal dotar a los progenitores de estrategias para la educación de sus hijos o hijas. Así, y en paralelo a las sesiones de los niños y las niñas en el subprograma Descubriéndonos, se llevan a cabo encuentros quincenales, centrados en poner en común la problemática de las familias y desarrollo de temas que ayudan a los progenitores a potenciar sus habilidades como educadores. De esta manera se abordan temáticas muy diversas, que van dirigidas al fomento de una educación en positivo, teniendo presentes técnicas de modificación de conducta (BORGES et al., 2016). No obstante, a las familias que permanecen en el PIPAC durante varios cursos los contenidos que se abordan les resultan repetitivos, manifestando la necesidad de seguir avanzando en la intervención. Por este motivo, en la XIII edición del PIPAC se incorpora un segundo nivel de formación, dirigiendo el foco de atención al trabajo de aspectos del desarrollo personal de los propios progenitores. Se detecta que éstos se habían olvidado por completo de ellos mismos y estaban volcados en su prole, en su educación, en responder a sus necesidades, etc. Sin embargo, poder desarrollar una adecuada labor como progenitor hace necesario prestar atención a su autocuidado ya que esto revertirá en una adecuada interacción con sus hijos e hijas. Así, se comienza favoreciendo un mayor conocimiento de sí mismos y facilitándoles estrategias para el adecuado control y la gestión efectiva de las emociones (RODRÍGUEZDORTA et al., 2016). Después de dos años abordando la gestión emocional, el trabajo con los progenitores se dirige a la gestión del estrés y la ansiedad. Más adelante, se incorporan otros aspectos, como la adolescencia o técnicas de estudio que les permitan contar con más recursos para dar respuesta a las demandas de sus hijos e hijas. Durante la presente edición, y dada la relevancia y los problemas que 
ha causado la pandemia de la COVID-19, se ha trabajado de forma muy intensa el estrés que ha supuesto este hecho.

En estos últimos años del programa y debido a que hay familias que llevan muchos años en él y que ya han trabajado los aspectos de los dos niveles mencionados, se crea un tercero donde el trabajo es más personal e individualizado ya que se trata de un grupo pequeño. Aquí, se plantean aspectos de gran relevancia para los participantes y que son abordados de forma más especializada. De esta manera, en la actualidad, Encuentros queda estructurado en tres niveles, nivel 1 formado por las familias que llevan menos de dos años en él, nivel 2 formado por las familias que llevan más de dos años en el programa y nivel 3 formado por las familias más veteranas.

Además, desde los inicios de Encuentros, se incluyen sesiones formativas conjuntas que son impartidas por expertos en diferentes disciplinas de especial interés para los progenitores, abordando temas diversos, como el acoso escolar, las drogas, la pandemia o la diversidad de género.

Otra de las cuestiones que se abordan desde el PIPAC, buscando la interacción entre participantes, familias y educadores, son las actividades complementarias. Están divididas en dos tipos: por un lado, las visitas educativas, siendo sus objetivos fundamentales cubrir los temas de interés de los participantes, fomentar las relaciones informales entre ellos y los educadores del programa, y cohesionar al grupo de familias. Por otro, los talleres y campamentos de verano (RODRÍGUEZ-DORTA, 2017). Los objetivos fundamentales de éstos son abordar en profundidad aspectos que durante el desarrollo del curso se han identificado como necesarios y trabajar también temas de interés para los participantes, todo ello desde un punto de vista más lúdico debido a la época vacacional.

Sin duda, los programas extraescolares son fundamentales para brindar una adecuada respuesta a las necesidades educativas del alumnado con Altas Capacidades Intelectuales. Su relevancia hace necesario no perder de vista la comprobación de que con ellos se alcanzan los objetivos previstos, siendo imprescindible llevar a cabo un adecuado sistema de evaluación que permita tanto comprobar esto, como la mejora y adaptación continua y si el programa da lugar a otros efectos que no se han tenido en cuenta (BORLAND, 2003).

Sin embargo, la evaluación adecuada de un programa tiene ciertas dificultades. Es preciso contar con instrumentos de comprobada bondad, puede resultar tediosa, provocar desmotivación en los participantes y ocupar gran parte del tiempo del programa. No cabe duda de que evaluar es importante, pero no debe convertirse en el único objetivo. Por tanto, el reto está en integrar procedimientos no intrusivos, que a la vez que forman parte del programa como actividades desarrolladas en el mismo, permitan evaluar.

El PIPAC es sometido a evaluación sistemática tanto sumativa como formativa, de forma anual e interna (BORGES et al., 2016; BORGES et al., 2018; RODRÍGUEZ-DORTA et al., 2017; RODRÍGUEZ-NAVEIRAS; BORGES, 2016). 
La evaluación formativa del programa está dirigida al análisis de la implementación del mismo, evaluando a los educadores y el proceso llevado a cabo, mientras que la evaluación sumativa pretende comprobar si se han alcanzado los objetivos establecidos al inicio del curso y si han tenido lugar otros efectos como la satisfacción de los participantes. Los procedimientos empleados para llevar a cabo la evaluación han ido modificándose y evolucionando a lo largo de las diferentes ediciones del programa.

Para llevar a cabo la evaluación formativa, tanto los escolares participantes y los progenitores como los educadores y educadoras evalúan cada sesión. Así, para todos se pasa el control de asistencia, todos los participantes (escolares y progenitores) responden al Cuestionario de evaluación de las sesiones, y educadores y educadoras al Cuestionario de autoevaluación de su intervención en la sesión. Además, a través del Protocolo de Observación de Funciones Docentes (RODRÍGUEZ-NAVEIRAS, 2011), se extraen los comportamientos más frecuentes y los patrones comportamentales significativos que los educadores llevan a cabo en las sesiones. Este último procedimiento de evaluación, dado que supone un gran costo en tiempo y recursos, se lleva a cabo de forma puntual, pero se ha utilizado en diversos trabajos fin de máster (GIL MARTÍNEZ, 2010; MARTÍN HERNÁNDEZ, 2010).

No obstante, tras detectar ciertas dificultades para cumplimentar el Cuestionario de evaluación de las sesiones en los participantes de menor edad (nivel 1), se plantea como alternativa al mismo para este grupo la actividad "Hoy me siento", permitiendo conocer cómo se sienten los participantes al inicio de la sesión y al final de la misma. Con respecto a la evaluación a través de metodología observacional, se integra también un nuevo instrumento para evaluar la interacción de los participantes en las sesiones, Protocolo de Observación de Interacción en el Aula (CADENAS; BORGES, 2016; HERNÁNDEZ LASTIRI, et al., 2019). Por otro lado, el Cuestionario de evaluación de las sesiones por parte de los progenitores pasa a un formato electrónico a través del programa Socrative, resultando a los padres y las madres más cómodo para su cumplimentación y permitiendo al equipo de trabajo del programa centralizar los datos obtenidos a través del mismo. Además, se incluye para los progenitores procedimientos de evaluación que combinan una actividad desarrollada en el programa con aspectos valorativos del mismo, "El Baúp" y "Ventana a la reflexión". La primera se desarrolla en el Nivel 1 y consiste en que los progenitores registran al inicio del curso aspectos positivos y negativos de su autoimagen como educadores y al final del mismo, de los aspectos trabajados, lo que han puesto en práctica, si les ha funcionado y lo que han conseguido. La segunda se lleva a cabo en el nivel 2 y a través de ella los progenitores registran, al final de curso, los datos de cuatro aspectos fundamentales, lo que han aprendido, las situaciones estresantes han vivido, qué han aplicado de lo aprendido, y de lo que han aplicado, qué les ha resultado útil.

Para los educadores también se incluyen algunas modificaciones. El Cuestionario de autoevaluación de su intervención en la sesión es también pasado a formato electrónico; se incluye el Cumplimiento de los indicadores de cada una de las actividades, a través del cual los/as educadores registran en qué grado se han cumplido los indicadores establecidos para cada sesión en cada una de las actividades desarrolladas, lo que permite 
establecer a su vez si se han alcanzado los objetivos marcados para cada actividad; y el PROFUNDO, $v 2$ es adaptado y actualizado dando lugar al PROFUNDO, v3 (AGUIRRE et al., 2017).

Para llevar a cabo la evaluación sumativa, concretamente para evaluar la eficacia del programa, tanto escolares participantes como progenitores responden al inicio y al final del curso a los siguientes cuestionarios: Cuestionario de expectativas y motivación del curso, Cuestionario sobre comportamientos personales y familiares y Sistema de evaluación de la conducta de niños y adolescentes (REYNOLDS; KAMPHAUS, 2004). Además, los escolares participantes responden también al Test Autoevaluativo Multifactorial de Adaptación Infantil (TAMAI) (HERNÁNDEZ, 1990) y los progenitores al Cuestionario de los conocimientos de los principios conductuales aplicados a los niños (O’DELL; TARLER-BENLOLO; FLYNN, 1979) y a una entrevista semiestructurada que se realiza al final del curso.

Sin embargo, debido la dificultad para responder a los cuestionarios de expectativas y motivación del curso y sobre comportamientos personales y familiares por parte de los niños y las niñas del nivel 1, éstos fueron adaptados sustituyendo los ítems por viñetas con dibujos. Una vez puesta su adaptación en práctica, nuevamente se detecta dificultad en este grupo para responderlos, ya que los participantes de este nivel seleccionan aquellas viñetas que contenían los dibujos que más les gusta y no las que se corresponden con su respuesta a la cuestión planteada. Por tanto, debido a la dificultad que continúan entrañando, dejan de pasarse al nivel 1. Para los escolares participantes del resto de niveles y para los progenitores, con el objetivo de facilitar y agilizar su aplicación, se reduce el número de ítems de estos cuestionarios. Además, para los progenitores pasan a un formato electrónico.

Por otra parte, los Cuestionarios BASC, TAMAI y el Cuestionario de los conocimientos de los principios conductuales aplicados a los niños, dejan de administrarse debido a su extensión y a lo tediosa que resulta su aplicación, y se incluye para los escolares participantes, salvo para el nivel 1, Evaluación de la Competencia Social y de la Interacción con los compañeros: Entrevista CEIC y CEICA (DÍAZ-AGUADO et al., 1995).

Para llevar a cabo la evaluación sumativa, concretamente el logro de otros efectos no previstos, esto es, la efectividad, tanto escolares participantes como progenitores responden al final del curso al Cuestionario de evaluación de educadores y al Cuestionario de evaluación del programa. Ambos cuestionarios entrañan dificultad a la hora de completarlos al llevar a confusión, puesto que se presentan como escala bipolar; el primero con siete alternativas de respuesta y 14 reactivos y el segundo con diez alternativas de respuesta y 18 reactivos. Por esta razón, son adaptados a cuestionarios con escala de respuesta tipo Likert, se dejan de aplicar al nivel y pasan a formato electrónico para los progenitores.

Como se ha visto, a lo largo de sus 18 años de andadura, el PIPAC se ha ido adaptando y evolucionando de forma continua con respecto a sus características y sistema de evaluación, lo que ha hecho que éste presente diversas fortalezas que favorecen su éxito y que, a continuación, se pasa a detallar. 


\section{Los aspectos más significativos del PIPAC}

Después del repaso hecho a las actividades y la forma de hacer en el programa, es importante sintetizar lo que supone, a nuestro entender, los aspectos más relevantes del mismo.

Un primer aspecto ha sido la forma de implementar el programa. Desde el inicio, uno de los aspectos que parecía de especial relevancia era evitar que las sesiones del programa recordaran el clima del aula. Por tanto, eso supone un reto esencial. Si bien cada sesión se diseña y programa de forma absolutamente estructurada, como se ha comentado, la realización de la misma se lleva a cabo de una forma lúdica: las actividades se plantean como un juego, de forma que los y las participantes aprendan divirtiéndose. Eso supone también una dificultad añadida: es preciso que puedan interaccionar y se diviertan, pero que haya la disciplina necesaria para poder desarrollar la sesión con normalidad. Eso ha supuesto unas de las dificultades que han tenido que enfrentar, con éxito, el equipo de educadores y educadoras.

En segundo lugar, en el PIPAC se ha contado siempre con al menos dos docentes por nivel, e incluso más. El o la educadora principal se encarga de llevar el timón de la sesión, mientras que el segundo, educador o educadora básico/a apoya al primero. Esa doble figura docente ha resultado ser de gran valor, porque permite dinamizar mucho más la sesión, si bien exige una clara coordinación entre docentes. Esto ha sido objeto de investigación (Aguirre et al., 2017), habiendo analizado la relación existente entre educadores como parte de la evaluación formativa del programa, para analizar posibles discrepancias, si las hubiera, y plantear soluciones.

Otro de los aciertos del programa ha sido incluir en él a toda la familia. Por una parte, se ha señalado la importancia de incorporar a los progenitores, pues juegan un papel fundamental en la educación de sus hijos e hijas, y es absolutamente necesario que sepan con claridad lo que son las altas capacidades intelectuales y tener claras directrices para el desarrollo de sus potencialidades. Pero en el programa también se han incorporado los hermanos y hermanas, siempre que sus edades coincidieran con las que se incluyen en los diversos niveles del PIPAC. Además de que se evitan etiquetas y discriminaciones, es bastante habitual, dado el gran componente genético, que sean prácticamente todos hijos o hijas de una familia de altas capacidades, por lo que se ven también favorecidos al participar. Además, ha sido bastante habitual que las familias decidan diagnosticar al hermano o hermana no nominado, encontrándose muy frecuentemente con su alta capacidad, que había pasado desapercibida.

El carácter integral del programa en cuanto a contenidos también se puede destacar como logro. Si bien como grupo no se puede decir que el alumnado de altas capacidades tenga desajuste personal o social, si es cierto que la participación en el programa, donde suelen encontrar mayor homogeneidad de intereses beneficia a este alumnado y les hace sentir más a gusto, e integrantes de un grupo de iguales. Por otra parte, el incluir un trabajo transversal que se desarrolla durante el trimestre, exige que busquen información y que aprendan a integrarla y a trabajar en grupo. Finalmente, tienen que desarrollar 
habilidades de exposición pública, para su presentación en la sesión de final de trimestre, en el que participan todos los miembros del programa, incluidos los progenitores, presentando los logros hechos durante el trimestre.

Por otra parte, el programa siempre ha tenido un interés formativo, incorporando alumnado de carreras relevantes para las altas capacidades (psicología, logopedia, trabajo social, educación, magisterio) como educadores en formación. Esto se ha hecho aceptando voluntarios, pero también a través de convenio con la ULL, ofertando la posibilidad de hacer prácticas externas de grado y máster. Esta formación permite paliar el escaso peso que los contenidos de altas capacidades tienen en los currículos de grado y máster de las disciplinas mencionadas. Además de intervenir en las sesiones del programa, esta formación se ha ido reglando y se estructura para que una la teoría con la práctica. Además, no solo se recibe alumnado de la ULL, sino que es frecuente tener en prácticas estudiantes americanos (fundamentalmente México y Brasil) o europeos (Portugal y Holanda), tanto de grado, como de máster y doctorado.

Ese carácter universal del PIPAC se pone de manifiesto también en la internacionalización del mismo, pues se imparte en diversos estados de México. En breve también se va a implementar en Brasil y, más cerca, en la isla vecina de Gran Canaria, con profesionales formados en el PIPAC.

Otro aspecto a destacar es la capacidad de adaptación a las realidades que vienen dadas. En ese sentido, la pandemia de la COVID-19 ha obligado a adaptarse a un formato online. En primer lugar, durante el confinamiento, que supuso no poder seguir con el programa presencial, hubo de transformarse de la noche a la mañana en formato online, sin una planificación previa, forzados por las condiciones del encierro. No obstante, y dada la alarma sanitaria, el PIPAC XVIII se está desarrollando de forma online, ya sí con una cuidadosa planificación. No ha sido fácil, porque hay que contemplar que las familias tengan disponibles los medios técnicos para poder llevar adelante la sesión, e incluso los materiales para implementar las actividades. Ha habido que incrementar la motivación, por ejemplo, con sesiones informales para apoyar la cohesión grupal, así como adaptar los contenidos al formato virtual. Una explicación más detallada se ha presentado en el congreso de CIVIT (ORTEGA et al., 2021).

\section{La respuesta de la ULL a las altas capacidades}

La Universidad de La Laguna, dando la importancia que merece a las altas capacidades, ha promovido diversas acciones dirigidas a este alumnado. En primer lugar, se cuenta con el Aula Cultural de Altas Capacidades Intelectuales ${ }^{1}$ donde se aglutina todo el conjunto de actividades sobre altas capacidades. Además, se implementan otros dos programas, uno de mentorías, COMPARTE CON LA ULL, donde se da una gran importancia a la incentivación de vocaciones científicas, pues los mentores

${ }^{1}$ (https://www.ull.es/portal/aulasycatedrasculturales/aula-cultural-altas-capacidades/). 
son el alumnado de los programas de doctorado, que difunden su tesis doctoral a estudiantes de altas capacidades de entre 6 y 16 años de edad (BORGES et al., 2018; TRUJILLO; AGUIRRE, 2019). El programa ATENEA-ULL (AGUIRRE et al., 2021; SILIÓ et al., 2020) está dirigido al alumnado universitario, se implementa este curso en su segunda edición. Se están realizando convenios con universidades europeas y americanas para internacionalizarlo.

Finalmente, en el aspecto de difusión, se cuenta también con un congreso, Jornadas Internacionales sobre Panorámica en Intervención en Altas Capacidades, que aglutina investigadores y expertos en el campo del talento y la alta capacidad intelectual, que este año se realizará en su quinta edición.

\section{REFERÊNCIAS}

AGUIRRE, Triana; BORGES, África; RODRÍGUEZ-DORTA, Manuela. Evolución del comportamiento de los educadores en función de su experiencia en un programa de Altas Capacidades, TALINCREA, v. 4, n. 2, p. 39-56, 2017. Disponível em: https://www.cucs.udg.mx/talineng/sites/default/files/adjuntos/04 07/07 Evolucion.pdf. Acesso em: 06 jul. 2021.

AGUIRRE, Triana Delagdo; RODRIGUEZ-DORTA, Manuela; HERNÁNDEZ, María José Dorta; BORGES, África. The ATENEA-ULL program: A proposal to increase the motivation of undergraduates, $A M A$ zônica, v. 13, n. 1, p. 360-379, 2021. Disponível em: https://periodicos.ufam.edu.br/index.php/amazonica/article/view/8327/5937. Acesso em: 06 jul. 2021.

ALENCAR, Eunice Maria Lima de. Dificultades socio-emocionales del alumnado con altas habilidades. Revista de Psicología, v. 26, n.1, p. 43-62, 2008. Disponível em:

http://pepsic.bvsalud.org/scielo.php?script=sci arttext\&pid=S0254-92472008000100004. Acesso em: 06 jul. 2021.

ARANCIBIA, Violeta; BOYANOVA, Diana; GONZÁLEZ, Pablo. Cognitive Characteristics of Gifted and Not Gifted Fifth-grade Chilean Students from Economically Vulnerable Contexts, Universal Journal of Educational Research, v. 4, n. 4, p. 744-754, 2016. Disponível em: https://doi.org/10.13189/ujer.2016.040411. Acesso em: 15 maio 2021.

ARETXAGA, Lorea; ALBES, Carmen; ETXEBARRIA, Izaskun; GALENDE, Isabel; SANTAMARÍA, Arantza; URIARTE, Belén; VIGO, Pedro. Orientaciones educativas. Alumnado con altas capacidades intelectuales. Servicio Central de Publicaciones del Gobierno Vasco, 2013. Disponível em: https://www.euskadi.eus/contenidos/documentacion/inn doc esc inclusiva/es def/adjuntos/escuel a-inclusiva/100012c Pub EJ altas capacidades c.pdf. Acesso em: 15 jun. 2020.

BORGES, África; HERNÁNDEZ-JORGE, Carmen. Gifted child in the family: early detection of giftedness, Talincrea, v. 4, n. 8, p. 40-48, 2018. Disponível em:

https://www.cucs.udg.mx/talineng/sites/default/files/adjuntos/04 08/07 Alta.pdf. Acesso em: 10 jun. 2020. 
BORGES, África; HERNÁNDEZ-JORGE, Carmen; RODRÍGUEZ-NAVEIRAS, Elena. La adaptación social de niños y niñas de altas capacidades intelectuales: Un acercamiento cualitativo, Sobredotaçao, v. 9, p. 119-130, 2008. Disponível em: https://www.researchgate.net/publication/308169698 Borges A Hernandez-Jorge C y RodriguezNaveiras E 2008. Acesso em: 07 jul. 2020.

BORGES, África; HERNÁNDEZ-JORGE, Carmen; RODRÍGUEZ-NAVEIRAS, Elena. Evidencias contra el mito de la inadaptación de las personas con altas capacidades intelectuales, Psicothema, v. 23, n. 3, p. 362-367, 2011. Disponível em: http://www.psicothema.com/pdf/3895.pdf. Acesso em: 07 jul. 2020.

BORGES, África; RODRIGUEZ-DORTA, Manuela; DELGADO, Triana Aguirre; HERNÁNDEZ DORTA, María José; GONZÁLEZ, Francisco José Noda. Evaluación de la Primera Edición del Programa de Mentorías Comparte con la Universidad de La Laguna (ULL), TALINCREA, v. 5, n. 9, p. 15-32, 2018. Disponível em:

https://www.cucs.udg.mx/talineng/sites/default/files/adjuntos/05 09/05 Evaluacion.pdf. Acesso em: 10 abr. 2020.

BORGES, África; RODRÍGUEZ-DORTA, Manuela; RODRÍGUEZ-NAVEIRAS, Elena. El programa Encuentros: La integración de los programas para progenitores en la intervención con alumnado de altas capacidades. AMAzônica, v. 23, n. 2, p. 213-236, 2016. Disponível em: https://periodicos.ufam.edu.br/index.php/amazonica/article/view/4684/3808. Acesso em: 21 mar. 2021.

BORGES, África; RODRÍGUEZ-NAVEIRAS, Elena; RODRÍGUEZ-DORTA, Manuela. Ajuste personal y social del alumnado de altas capacidades: Evidencias empíricas y respuesta educativa. In: PIKE, Fernanda Hellen Ribeiro et al. (Orgs.). Educação De Superdotados e talentosos. Emoção e Criatividade. Curitiba: Juruá, 2018, p. 43-60.

BORLAND, James. Evaluating gifted programs: A broader perspective. In: COLANGELO, Nicholas; DAVIS, Gary. (Eds.). Handbook of gifted education, 3.ed. Boston: Allyn and Bacon, 2003, p. 293-307.

CADENAS, María; BORGES, África. Procedimientos e instrumentos de evaluación para el estudio de la interacción social. España: Editorial Académica Española, 2016.

CADENAS, María; DÍAZ, P.; HERNÁNDEZ, P.; HERRANZ, N.; MARÍN, M.; PÉREZ, J.; RODRÍGUEZ-DORTA; Manuela; SANCHO, Daniel. La acción social como objetivo del trabajo colaborativo en el Programa Integral para Altas Capacidades. Comunicación presentada en las Jornadas Internacionales sobre panorámica de intervención en altas capacidades intelectuales, 3. ed., San Cristóbal de La Laguna, España, 2017.

COMES, Gabriel; DÍAZ, Elena; DE LA ROSA, Antonio Luque; MOLINER, Odete. La evaluación psicopedagógica del alumnado con altas capacidades intelectuales, Revista Educación Inclusiva, v. 1, n. 1, p. 103-117, 2008. Disponível em:

https://revistaeducacioninclusiva.es/index.php/REI/article/view/23/22. Acesso em: 15 jun. 2021.

COMES, Gabriel; DÍAZ, Elena; DE LA ROSA, Antonio Luque; TUDELA, Juana María Ortega. Analysis of the Spanish law on the education of highly gifted students. EA, Escuela abierta: revista de Investigación Educativa, v. 12, p. 9-31, 2009. Disponível em:

http:/ / dialnet.unirioja.es $/$ servlet/articulo? codigo $=3277694 \&$ info $=$ resumen\&idioma $=$ SPA. Acesso em: 15 jun. 2021. 
CURBELO, Sheila; MONTERO, Rubens. El trabajo colaborativo para el trabajo grupal y la creatividad en un programa extraescolar en altas capacidades (PIPAC): el arte. TALINCREA, v. 6, n. 12, p. 3-19, 2020. Disponível em:

https://www.cucs.udg.mx/talineng/sites/default/files/adjuntos/06 12/04 trabajo.pdf. Acesso em: 10 fev. 2021.

DÍAZ-AGUADO, María José; ROYO GARCIA, Pilar; MARTÍNEZ, Rosario. Instrumentos para evaluar la integración escolar. España: GRÁFICAS JUMA, 1995.

DÍAZ CABRERA, Lourdes. El mundo del Superdotado: los compañeros de aula [Tesis de licenciatura]. Didáctica e Investigación Educativa y del Comportamiento. Universidad de La Laguna, 2004.

FLORES, Juan Francisco; VALADEZ, María de los Dolores; BORGES, África. Efectos de un programa de orientación a padres de hijos con altas capacidades. TALINCREA, v. 7, n. 13, p. 33-47, 2020. Disponível em:

https://www.cucs.udg.mx/talineng/sites/default/files/adjuntos/07 13/06 Efectos.pdf. Acesso em: 31 jun. 2021.

GAGNÉ, Françoys. Building gifts into talents: Detailed overview of the DMGT 2.0. In:

MACFARLANE, Bronwyn; STAMBAUGH, Tamra (Eds.). Leading change in gifted education: The festschrift of Dr. Joyce VanTassel-Baska, Texas: Prufrock Press, 2009, p. 61-80.

GIL MARTÍNEZ, Déborah. Profundo: Protocolo de Observación de Funciones Docentes. Depuración del instrumento, 2010. [Trabajo de fin de Máster]. Máster Universitario en Intervención y Mediación Familiar por las Universidades de La Laguna y Las Palmas de Gran Canaria, Universidad de La Laguna.

GÓMEZ MADRID, Marina. Estudio sobre la eficacia de un programa para la mejora de la tolerancia a la frustración en alumnado con altas capacidades [Trabajo de Fin de Máster]. Departamento de Psicología Clínica, Psicobiología y Metodología. Universidad de La Laguna, 2020. Disponível em: https://riull.ull.es/xmlui/handle/915/23383. Acesso em: 15 jun. 2021.

HERNÁNDEZ, Pedro. (1990). Test Evaluativo Multifactorial de Adaptación Infantil (T.A.M.A.I.). Madrid: TEA Ediciones.

HERNÁNDEZ-LASTIRI, Pablo; BORGES, África y CADENAS, María. Differences in Mechanisms of Social Interaction of Gifted Children Based on Peer Acceptance, Acción Psicológica, v. 16, n. 1, p. 13-30, 2019. Disponível em; https://doi.org/10.5944/ap.16.1.22169. Acesso em:_15 jun. 2021.

HERNÁNDEZ-TORRANO, Daniel; GUTIÉRREZ SÁNCHEZ, Marta. El estudio de la alta capacidad intelectual en España: Análisis de la situación actual, Revista de Educación, n. 364, p. 251-272, 2014. Disponível em: DOI: 10.4438/1988-592X-RE-2014-364-261. Acesso em: 23 jul. 2021.

MARÍN, Mariana; RODRÍGUEZ, Silvia; BORGES, África. Mejora de la caligrafía en un programa integral para altas capacidades, TALINCREA, v. 6, n. 12, p. 77-88, 2020. Disponível em: http://www.talincrea.cucs.udg.mx//sites/default/files/adjuntos/06 12/09 Mejora.pdf. Acesso em: 12 jun. 2021.

MARTÍN HERNÁNDEZ, Jonatan. Evaluación de proceso: análisis del incremento de la participación de la monitora en función de la experiencia, 2010. [Trabajo de Fin de Máster]. Máster Universitario en Intervención y Mediación Familiar por las Universidades de La Laguna y Las Palmas de Gran Canaria, Universidad de La Laguna. 
MATÍAS RANCEL, Raquel. Relaciones interpersonales en niños y adolescentes de altas capacidades intelectuales con sus pares. TALINCREA, v. 7, n. 13, p.72-80, 2020. Disponível em: https://www.cucs.udg.mx/talineng/sites/default/files/adjuntos/07 13/08 Relaciones.pdf. Acesso em: 06 jan. 2021.

O`DELL, Stan L.; TARLER-BENLOLO, Linda; FLYNN, John. An instrument to measure knowledge of behavioral principles as applied to children. Journal of Behavior Therapy and Experimental Psychiatry, v. 10, p. 29-34, 1979. Disponível em: https://doi.org/10.1016/0005-7916(79)90033-8. Acesso em: 10 out. 2019.

OLSZEWSKI-KUBILIUS, Paula M.; KULIEKE, Marilynn; KRASNEY, Noma. Personality dimensions of gifted adolescents: a review of the empirical literature. Gifted Child Quarterly, v. 32, n. 4, p. 347-352, 1988. Disponível em: https://doi.org/10.1177/001698628803200403. Acesso em: 10 out. 2019.

ORTEGA, Luis; GÓMEZ, Marina; PAILLET, Alicia. Programa de intervención socioafectiva para niños y niñas de altas capacidades en formato online [Comunicación] CIVIT, 2021.

PEÑAS, María. Características socioemocionales de las personas adolescentes superdotadas. Ajuste psicológico y negación de la superdotación en el concepto de si mismas. Secretaría General Técnica, 2006. Disponível em:

https://sede.educacion.gob.es/publiventa/PdfServlet?pdf=VP12521.pdf\&area=E. Acesso em: 30 set. 2019.

PÉREZ, Luz. Superdotación y familia. Faísca, v.11, p. 17-36, 2004. Disponível em: file:///C:/Users/Windows\%2010/Downloads/Dialnet-SuperdotacionYFamilia-2476401.pdf. Acesso em: 17 ago. 2019.

PÉREZ, Josué; APERRIBAI, Leire; CORTABARRÍA, Lorea; BORGES, África. Examining the most and least changeable elements of the social representation of giftedness. Sustainability, v. 12, n. 13, p. 5361, 2020. Disponível em: https://doi.org/10.3390/su12135361. Acesso em: 19 mar. 2021.

PÉREZ HERNÁNDEZ, Niobe; RODRÍGUEZ-NAVEIRAS, Elena. Programa de intervención para la mejora de la tolerancia a la frustración en niños con altas capacidades. TALINCRE $A$, v. 6, n.11, p. 86-114, 2019. Disponível em:

http://www.talincrea.cucs.udg.mx//sites/default/files/adjuntos/06 11/10 Programa.pdf. Acesso em: 10 fev. 2020.

QUINTERO, Ricardo; GUTIÉRREZ, Samuel; BORGES, África. Mappeo de la identificación del Talento y las Altas Capacidades Intelectuales en España. Revista AMAzônica, v. 26, n. 2, p. 151-168, 2021. Disponível em:

https://www.periodicos.ufam.edu.br/index.php/amazonica/article/view/9040/6519. Acesso em: 16 ago. 2021.

REYNOLDS, Cecil; KAMPHAUS, Randy. BASC: Sistema de evaluación de la conducta de niños y adolescentes. TEA Ediciones, 2004.

RODRÍGUEZ-DORTA, Manuela. Talleres de verano del Programa Integral de Altas Capacidades. Comunicación presentada en las Jornadas Internacionales sobre panorámica de intervención en altas capacidades intelectuales, 2017, San Cristóbal de La Laguna, España.

RODRÍGUEZ-DORTA, Manuela; BORGES, África; DÍAZ, M.; RODRÍGUEZ-NAVEIRAS, Elena. Controly manejo de emociones. Un programa para padres de alumnado con altas capacidades intelectuales. 
Comunicación presentada en el Congresso Internacional ANEIS: Saberes Consilidados e Desenvolvimentos Promissores, 2017, Coimbra, Portugal.

RODRÍGUEZ-DORTA, Manuela; RODRÍGUEZ-NAVEIRAS, Elena; BORGES, África. Los programas para progenitores: evaluación de los dos niveles del Programa Encuentros. Comunicación presentada en las Jornadas Internacionales sobre panorámica de intervención en altas capacidades intelectuales, 2017, San Cristóbal de La Laguna, España.

RODRÍGUEZ NAVEIRAS, Elena. Adaptación sociopersonal de adolescentes de altas capacidades no identificados [Memoria para el Diploma de Estudios Avanzados], 2006. Didáctica e Investigación Educativa y del Comportamiento. Universidad de La Laguna.

RODRÍGUEZ-NAVEIRAS, Elena. PROFUNDO: Un instrumento para la evaluación de proceso de un programa de altas capacidades. Tesis Doctoral. Universidad de La Laguna. Soportes Audiovisuales e informáticos, 2011. Disponível em:

https://riull.ull.es/xmlui/bitstream/handle/915/9771/cs265.pdf?sequence=1\&isAllowed=y. Acesso 14 maio 2019.

RODRÍGUEZ-NAVEIRAS, Elena; BORGES, África. Evaluación sumativa y formativa del programa integral para altas capacidades. In: VALADEZ, María de los Dolores et al. (Eds.), Programas de intervención para niños con altas capacidades y su evaluación. México: Manual Moderno, 2016, p. 49-65.

RODRÍGUEZ-NAVEIRAS, Elena; BORGES, África. Programas extraescolares: una alternativa a la respuesta educativa de altas capacidades. Revista de Educación y Desarrollo, v. 52, p. 19-27, 2020.

Disponível em:

https://www.cucs.udg.mx/revistas/edu desarrollo/anteriores/52/52 RodriguezBorges.pdf. Acesso em: 14 jul. 2021.

RODRÍGUEZ-NAVEIRAS, Elena et al. Educational responses to students with high abilities from the parental perspective. Frontiers in psychology, v. 10, n. 1187, 2019. Disponível em: https://scihub.se/https://dx.doi.org/10.3389\%2Ffpsyg.2019.01187. Acesso em: 16 maio 2020.

RODRÍGUEZ-NAVEIRAS, Elena et al. Programa integral para altas capacidades "Descubriéndonos": una guía práctica de aplicación. Manual Moderno, 2015.

RODRÍGUEZ-NAVEIRAS, Elena et al. Differences in working memory between gifted or talented students and community samples: A meta-analysis, Psicothema, v. 31, n. 3, p. 255-262, 2019. Disponível em: https://sci-hub.se/https://doi.org/10.7334/psicothema2019.18. Acesso em: 7 abr. 2020.

SANCHO, Daniel et al. El trabajo colaborativo a través de proyectos final de trimestre. Comunicación presentada en las Jornadas Internacionales sobre panorámica de intervención en altas capacidades intelectuales, 2017, San Cristóbal de La Laguna, España.

SASTRE-RIBA, Sylvia. Niños con altas capacidades y su funcionamiento cognitivo diferencial. Revista de Neurología, v. 46, n.1, p. 11-16, 2008. Disponível em:

https://sid.usal.es/idocs/F8/ART13977/ni\%C3\%B1os con altas capacidades y su funcionamiento. pdf. Acesso em: 11 jun. 2018.

SASTRE-RIBA, Sylvia. Alta capacidad intelectual: perfeccionismo y regulación metacognitiva. Revista de Neurología, v. 54, n. 1, p. 21-29, 2012. Disponível em:

http://www.webdocente.altascapacidades.es/Modulo-3-Diagnostico/perfeccionismo.pdf. Acesso em: 19 jul. 2020. 
SILIÓ RODRÍGUEZ, María et al. Concepción que el profesorado y el alumnado participante en el programa de mentoría ATENEA-ULL tienen de las altas capacidades intelectuales, TALINCREA, v. 6, n. 12, p. 89-102, 2020. Disponível em:

https://www.cucs.udg.mx/talineng/sites/default/files/adjuntos/06 12/10 Concepcion.pdf. Acesso em: 18 ago. 2020.

STEINER, Hillary; CARR, Martha. Cognitive development in gifted children: Toward a more precise understanding of emerging differences in intelligence. Educational Psychology Review, v. 15, n. 3, p. $215-$ 246, 2003. Disponível em: https://sci-hub.se/10.1023/A:1024636317011. Acesso em: 18 out. 2020.

STOEGER, Heidrun et al. What is more important for fourth-grade primary school students for transforming their potential into achievement: the individual or the environmental box in multidimensional conceptions of giftedness? High Ability Studies, v. 25, n. 1, 5-21, 2014. Disponível em: https://doi.org/10.1080/13598139.2014.914381. Acesso em: 10 nov. 2018.

TANNENBAUM, Abraham. Giftedness: A psychosocial Approach. In: STENRBERG, Robert; DAVISON, Janet (Eds.). Conceptions of giftedness. Cambridge University Press., 1986, P. 21-52.

TRUJILLO, Jorge Andrés; AGUIRRE, Triana. Evaluación de la Segunda Edición del Programa de Mentorías Comparte con la Universidad de La Laguna (ULL). TALINCREA, v. 6, n. 11, P. 43-61, 2019. Disponível em:

https://www.cucs.udg.mx/talineng/sites/default/files/adjuntos/06 11/07 Evaluacion.pdf. Acesso em: 15 maio 2020.

VAIVRE-DOURET, Laurence. Developmental and Cognitive Characteristics of "High-Level Potentialities" (Highly Gifted) Children. International Journal of Pediatrics, p.1-14, 2011. Disponível em: https://doi.org/10.1155/2011/420297. Acesso em: 7 out. 2019.

VAN BOXTEL, Herman; MÖNKS, Franz. Los adolescentes superdotados: una perspectiva evolutiva. In: FREEMAN, Joan (Ed.). Los niños superdotados. aspectos psicológicos y pedagógico. España: Santillana, 1988, p. 306-327.

Recebido em: 19 de abril de 2021. Aprovado em: 11 de setembro de 2021. 